\title{
The Feasibility of Monoculture and Polyculture of Striped Catfish and Nile Tilapia in Different Proportions and Their Effects on Growth Performance, Productivity, and Financial Revenue
}

\author{
Abdallah Tageldein Mansour 1,2,*(D), Belal Wagih Allam ${ }^{2, *}$, Tarek Mohamed Srour ${ }^{2}$, Eglal Ali Omar ${ }^{2}$, \\ Abdel Aziz Mousa Nour ${ }^{3}$ and Hala Saber Khalil ${ }^{4,5, *(D)}$ \\ 1 Fish and Animal Production and Aquaculture Department, College of Agriculture and Food Sciences, \\ King Faisal University, P.O. Box 420, Al-Ahsa 31982, Saudi Arabia \\ 2 Fish and Animal Production Department, Faculty of Agriculture (Saba Basha), Alexandria University, \\ Alexandria 21531, Egypt; tarek-srour@alexu.edu.eg (T.M.S.); eglalomar@gmail.com (E.A.O.) \\ 3 Faculty of Agriculture, Department of Fish and Animal Production, Alexandria University, \\ Alexandria 21545, Egypt; nouraziz47@gmail.com \\ 4 WorldFish Center, Africa Aquaculture Research and Training Center, Abbassa 44662, Egypt \\ 5 National Institute of Oceanography and Fisheries (NIOF), Cairo 11516, Egypt \\ * Correspondence: amansour@kfu.edusa (A.T.M.); belal.w.allam@gmail.com (B.W.A.); \\ halasaber2011@yahoo.com (H.S.K.)
}

Citation: Mansour, A.T.; Allam, B.W.; Srour, T.M.; Omar, E.A.; Nour, A.A.M.; Khalil, H.S. The Feasibility of Monoculture and Polyculture of Striped Catfish and Nile Tilapia in Different Proportions and Their Effects on Growth Performance, Productivity, and Financial Revenue. J. Mar. Sci. Eng. 2021, 9, 586. https:// doi.org/10.3390/jmse9060586

Academic Editor: Francesco Tiralongo

Received: 15 April 2021

Accepted: 27 May 2021

Published: 28 May 2021

Publisher's Note: MDPI stays neutral with regard to jurisdictional claims in published maps and institutional affiliations.

Copyright: (C) 2021 by the authors Licensee MDPI, Basel, Switzerland. This article is an open access article distributed under the terms and conditions of the Creative Commons Attribution (CC BY) license (https:// creativecommons.org/licenses/by/ $4.0 /)$.
Abstract: Cultivation of species of high growth rates is a key achievement of sustainable aquaculture development, with the aim of increasing animal protein per capita, maintaining food security and preserving freshwater usage. The present study was conducted to evaluate the feasibility of monoculture and polyculture of striped catfish, Pangasianodon hypophthalmus, and Nile tilapia, Oreochromis niloticus, in different proportions and their effect on growth performance, survival, productivity, feed utilization, body composition, and financial revenue. Five experimental treatments were designed as follows: monoculture of striped catfish $(100 \%)$, Nile tilapia $(100 \%)$ and polyculture in different proportions of striped catfish and Nile tilapia ( $25 \%: 75 \% ; 50 \%: 50 \% ; 75 \%: 25 \%$, respectively), each in three replicates. The fish feeding regime consisted of isonitrogenous $\left(307.80 \mathrm{~g} \mathrm{~kg}^{-1}\right)$ and isocaloric $\left(19.27 \mathrm{~kJ} \mathrm{~g}^{-1}\right)$ diets for 14 weeks. The results revealed that the highest growth performance, feed utilization, survival of striped catfish were obtained in monoculture, followed by polyculture of striped catfish and Nile tilapia (in low proportions) (75\%:25\%). The total production per $\mathrm{m}^{3}$ reached $5.41 \mathrm{~kg} \mathrm{~m}^{-3}$ in the monoculture of striped catfish, this production decreased in polyculture by $52 \%$, $46 \%$ and $23 \%$ with $25 \% ; 50 \% ; 75 \%$ of striped catfish. The gross margin significantly increased, in case of striped catfish farmed in monoculture, compared to other polyculture proportions. While the gross margin per $\mathrm{m}^{-3}$ of water was $6,0.5,1$ and $3 \$$ in monoculture and different polyculture proportions, respectively. Moreover, the feed cost per kilogram of protein gain significantly decreased in the monoculture of striped catfish compared to other polyculture treatments. The growth performance and survival of tilapia showed no significant difference among different treatments. In addition, the proximate chemical composition did not differ in respect to species in different studied treatments. Monoculture of striped catfish is recommended to achieve high production and improve financial revenue per water unit $\left(\mathrm{m}^{-3}\right)$, for better sustainable development of aquaculture.

Keywords: sustainable aquaculture; striped catfish; Nile tilapia; polyculture proportions; production; financial assessment

\section{Introduction}

Freshwater aquaculture significantly contributes in global fisheries production. The aquaculture production in Africa showed significant increase, compared to capture fisheries, reaching $\approx 2$ million tons in 2018 [1]. In most developing countries, the aquaculture industry 
plays an essential role in food security, providing a high quality animal protein source $[2,3]$. Nile tilapia is the main aquaculture indigenous species in Africa, it shows acceptable growth, feed efficiency and high marketability [4]. However, during the last few years, the production of Nile tilapia in Africa and Egypt, in particular, being one of the main tilapia producers, has been facing certain challenges, especially with the summer mortality phenomenon and freshwater shortage [5,6]. In addition, climate change and freshwater shortage have been a challenge these regions faced, which forced scientists to look for more sustainable solutions to maximize the aquaculture production and the efficient use of water unit [7]. Farming high growth rate species with high stocking density has revealed itself as a probable solution. New emerging fish species with high growth rate and intensification ability called striped catfish (Pangasianodon Hypophthalmus) started in Asia for several decades and commenced to be used in production trials in Egypt [8]. The growth performance of striped catfish reported to be higher than tilapia, accordingly, introducing striped catfish as new species in the farmed commodities besides Nile tilapia has become an idea considered interesting [8-10].

Striped catfish, P. Hypophthalmus, is one of the fast growth freshwater species incorporated in Asian aquaculture commodities starting from Mekong Delta of Vietnam and spread over Asian countries [11]. Striped catfish production markedly increased by $200 \%$ within the last decade, reaching $2359.5 \mathrm{~K}$ tons in 2018, ranking fourth in world production after carp, Nile tilapia, Atlantic salmon species [1]. Striped catfish has a fast growth rate, high feed conversion efficiency, accepted low to moderate protein levels, is cultured in high stocking density and resist several common aquaculture diseases [12].

The polyculture of striped catfish with different fish species was extensively investigated. The culture of striped catfish with carps was successful in the semi-intensive culture at 30:70 percent ratio [13]. The proportion of 1:1 of striped catfish and silver carp, Hypophthalmichthys molitrix, maintained acceptable growth performance and survival of both species, and economic benefit [14,15]. In addition, striped catfish production in polyculture with other species including catla, carp and freshwater prawn reached 5.88 ton hat $^{-1}$ and resulted in more profit than the monoculture of pangasius [16]. The polyculture of different aquaculture species has socioeconomic importance, as it introduces several products with different customer appeal, maximizes the efficient use of water body, controls fish recruits, and improves profitability $[9,17,18]$. On the other hand, a detrimental factor in setting up a polyculture system is identifying the optimal species ratio to maximize valorization of different ecological strata and water body, and considering species interaction (inter and intraspecific competition) $[18,19]$.

In addition, striped catfish and Nile tilapia are important species in global aquaculture in freshwater fish [20] and both may be cultured efficiently in high density and have similar feeding requirement $[8,21]$. The production of both species expected to reach $5-7.9 \mathrm{M}$ tons per year in 2030 [20].

Therefore, transition to intensive co-farming of both species has many benefits, including higher production, efficient water use and employment opportunities creation (higher on-farm wage, feed mills, inputs trading (e.g., feed and chemicals), fish harvesting, transportation and marketing), and support of a wide range of value chain actors [22,23]. In Egypt, we have imported striped catfish as ornamental fish, since 2018, we have succeeded in establishing its hatching technology and farming this kind of fish for human consumption. The main aquaculture system of striped catfish was polyculture with tilapia, as it has been proven that it lives in a symbiotic environment with tilapia. However, no optimal ratio of polyculture has been completely evaluated or validated in rearing stage. Thus, it is vital to assess the production and economic value of striped catfish and Nile tilapia culture for an informed farmer decision making regarding opportunities of co-farming of both species. Therefore, the objective of this study was to valorize the feasibility of monoculture and polyculture proportions of striped catfish (P. hypophthalmus) and Nile tilapia (O. niloticus) and their effect on growth performance, feed utilization, proximate composition and economic efficiency in rearing stage. 


\section{Materials and Methods}

\subsection{Experimental Fish and Facilities}

A total of three hundred striped catfish, P. hypophthalmus, fingerlings (14.5 \pm $0.17 \mathrm{~g} \mathrm{fish}^{-1}$ ) obtained from a private commercial freshwater fish farm located in Kilo-21, Alexandria-Matrouh Road, Alexandria, Egypt and of three hundred Nile Tilapia, O. niloticus, fingerlings $\left(14.5 \pm 0.15 \mathrm{~g}_{\text {fish }}{ }^{-1}\right)$ were obtained from a private commercial freshwater fish farm sited in Motobas, Kafr El Sheikh Governorate, Egypt. Before commencing the experiment, fish were acclimatized to laboratory conditions in circular fiberglass tanks (of $1 \mathrm{~m}^{3}$ ) for 14 days and fed basal diet at the Fish Nutrition Laboratory, Department of Animal and Fish Production, Faculty of Agriculture (Saba Basha), Alexandria University.

After acclimatization, the fish were randomly distributed into twelve circular fiberglass tanks (of $1 \mathrm{~m}^{3}$, filled with $800 \mathrm{~L}$ of water), at a stocking density of 40 fish per tank (50 fish $/ \mathrm{m}^{3}$ ). The daily water exchange rate was $30 \%$ and excreta were removed by manual siphoning. The rearing conditions were measured once daily at 9 a.m. as follows: water temperature $\left(27.19 \pm 3.2{ }^{\circ} \mathrm{C}\right)$, dissolved oxygen $\left(6.59 \pm 2.20 \mathrm{mg} \mathrm{L}^{-1}\right)$, using a portable DO meter (Crison, model OXI 45 P, Alella, Barcelona, Spain). pH (8.1 \pm 0.4$)$ and salinity $\left(0.2 \pm 0.4 \mathrm{~g} \mathrm{~L}^{-1}\right)$ were measured using an automatic probe, digital multimeter (Crison, model MM41, Alella, Barcelona, Spain). Total ammonia nitrogen $\left(0.005 \pm 0.002 \mathrm{~g} \mathrm{~L}^{-1}\right)$ was measured by HACH test kits, Model NI-12 the photoperiod regime was (8:16 h, light:dark) [24].

\subsection{Experimental Design and Diets}

The four experimental treatments of monoculture and polyculture proportions of striped catfish and Nile tilapia were applied in a complete random block design in triplicate tanks, as follows:

Treatment one $\left(\mathrm{T}_{1}\right)$ : Monoculture (100\% striped catfish).

Treatment two $\left(\mathrm{T}_{2}\right)$ : Polyculture (25\% striped catfish and $75 \%$ Nile tilapia).

Treatment three $\left(\mathrm{T}_{3}\right)$ : Polyculture (50\% striped catfish and 50\% Nile tilapia).

Treatment four $\left(\mathrm{T}_{4}\right)$ : Polyculture (75\% striped catfish and 25\% Nile tilapia).

Treatment five (T5): Monoculture (100\% Nile tilapia).

The fish were fed a basal diet (30.78\% crude protein and $19.27 \mathrm{~kJ} \mathrm{~g}^{-1}$ gross energy) as a predetermined requirement of omnivores fish in our laboratory [8]. Fish were fed three times a day at 9:00 a.m., 12:00 p.m. and 3:00 p.m., with feeding rate of 5\% in the first four weeks, $4 \%$ in the second four weeks and 3\% until the end of the experiment period for fourteen weeks. The introduced feed adjusted weekly according to changes in body weight and respected percent.

The experimental diet was prepared by grinding and mixing all ingredients with vitamins and minerals. Warm distilled water $\left(35^{\circ} \mathrm{C}\right)$ was added slowly until the diets began clumping, then pelleted by mill machine and dried using a forced air oven before being stored in plastic containers at $\left(20^{\circ} \mathrm{C}\right)$. The resulting pellet size was of $1.5 \mathrm{~mm}$. (cf. Table 1 for Diet formulation and proximate composition). Institutional Animal Care and Use Committee of Alexandria University (IACUC) approved experimental protocols (Approval No AU: 14200721310).

\subsection{Measured Parameters}

\subsubsection{Growth Performance and Feed Utilization Parameters}

The individual final body weight (FBW) of each experimental treatment was determined by dividing the total fish weight in each tank by the number of fish. The weight gain (WG), specific growth rate (SGR), survival percentage (\%) [25], feed conversion ratio (FCR), and protein efficiency ratio (PER) were calculated using the following equations:

$$
\mathrm{WG}\left(\mathrm{g} \mathrm{fish}^{-1}\right)=\mathrm{W}_{2}-\mathrm{W}_{1} \text {. }
$$


where $\mathrm{W}_{1}$ : initial weight of the fish $(\mathrm{g})$, and $\mathrm{W}_{2}$ : FBW of fish $(\mathrm{g})$.

$$
\operatorname{SGR}\left(\% \text { day }^{-1}\right)=100 \times\left(\ln W_{2}-\ln W_{1}\right) / \text { days. }
$$

where $\ln$ is the natural log.

$$
\begin{aligned}
& \text { Survival }(\%)=100 \times(\text { final number of fish/initial number of fish }) \\
& \text { Condition factor }(\text { K value })=100 \times\left[\text { fish weight }(\mathrm{g}) / \text { fish length }^{3}(\mathrm{~cm})\right]
\end{aligned}
$$

Production $\left(\mathrm{kg} \mathrm{m}^{-3}\right)=$ number of survived fish $\left(\mathrm{m}^{-3}\right) \times$ final stock density $\left(\mathrm{kg} \mathrm{fish}^{-1}\right)$

Total production $\left(\mathrm{kg} \mathrm{m}^{-3}\right)=$ production of striped catfish $\left(\mathrm{kg} \mathrm{m}^{-3}\right)+$ production of Nile tilapia $\left(\mathrm{kg} \mathrm{m}^{-3}\right)$.

$$
\begin{gathered}
\mathrm{FCR}=\text { feed intake }(\mathrm{g}) / \text { weight gain }(\mathrm{g}) . \\
\text { PER }=\mathrm{WG}(\mathrm{g}) / \text { protein intake }(\mathrm{g}) .
\end{gathered}
$$

Table 1. Formulation and proximate composition of experimental diet $\left(\mathrm{g} \mathrm{kg}^{-1}\right)$.

\begin{tabular}{ll}
\hline Ingredients & $\mathbf{g ~ k g}^{-\mathbf{1}}$ Diet \\
\hline Fish meal $(62 \% \mathrm{CP})$ & 150 \\
Soybean meal $(48 \% \mathrm{CP})$ & 300 \\
Corn gluten meal $(60 \% \mathrm{CP})$ & 100 \\
Yellow corn meal & 250 \\
Wheat bran & 110 \\
Wheat flour & 50 \\
Sunflower oil & 15 \\
Dicalcium Phosphate $^{1}$ & 5 \\
Vitamins mixture $^{1}$ & 10 \\
Minerals mixture $^{2}$ & 10 \\
\hline
\end{tabular}

\begin{tabular}{ll}
\hline \multicolumn{2}{l}{ Proximate chemical analysis $\left(\mathbf{g ~ k g}^{-\mathbf{1}}\right.$ dry matter) } \\
\hline Dry matter & 931.00 \\
Crude protein (CP) & 307.80 \\
Ether extract (EE) & 69.30 \\
Crude fiber (CF) & 17.80 \\
Ash & 65.90 \\
Nitrogen free extract (NFE) & 3 \\
Gross energy (GE; $\left.\mathrm{kJ} \mathrm{g}^{-1}\right)^{4}$ & 539.20 \\
\hline
\end{tabular}

${ }^{1}$ Vitamin premix (mg kg ${ }^{-1}$ diet): retinyl palmitate, $60 \mathrm{mg}$; cholecalciferol, $10 \mathrm{mg}$; DL-a-tocopherol acetate, $100 \mathrm{mg}$; menadione, $40 \mathrm{mg}$; thiamine- $\mathrm{HCl}, 25 \mathrm{mg}$; riboflavin, $25 \mathrm{mg}$; D-calcium pantothenate, $80 \mathrm{mg}$; pyridoxine- $\mathrm{HCl}$, $20 \mathrm{mg}$; meso-inositol, $1000 \mathrm{mg}$; D-biotin, $40 \mathrm{mg}$; folic acid, $7.5 \mathrm{mg}$; para-aminobenzoic acid, $25 \mathrm{mg}$; niacin, $100 \mathrm{mg}$; cyanocobalamin, $0.05 \mathrm{mg} .{ }^{2}$ Minerals premix (mg kg${ }^{-1}$ diet): $\mathrm{MnSO}_{4}, 10 \mathrm{mg}$; $\mathrm{MgSO}_{4}, 10 \mathrm{mg} ; \mathrm{KCl}, 95 \mathrm{mg}$; $\mathrm{ZnSO}_{4} \cdot 7 \mathrm{H}_{2} \mathrm{O}, 0.55 ; \mathrm{NaCl}, 165 \mathrm{mg} ; \mathrm{KI}, 1.0 \mathrm{mg} ; \mathrm{CuSO}_{4}, 12.5 \mathrm{mg} ; \mathrm{FeSO}_{4}, 105 \mathrm{mg} ; \mathrm{Co}, 1.5 \mathrm{mg} ; \mathrm{Na}_{2} \mathrm{SeO}_{3}, 1.0 \mathrm{mg}$. ${ }^{3} \mathrm{NFE}=100-(\mathrm{CP}+\mathrm{EE}+\mathrm{CF}+\mathrm{Ash}) .{ }^{4} \mathrm{GE}$ calculated on the basis of 23.6, 39.4 and $17.2 \mathrm{~kJ} \mathrm{GE} \mathrm{g}^{-1}$ protein, EE and carbohydrates, respectively [26].

\subsubsection{Financial Assessment}

The comparative financial assessment includes production costs, gross margin on seed and feed inputs in striped catfish production in regimes of monoculture and of polyculture proportions with Nile tilapia in water unit $\left(\mathrm{m}^{3}\right)$. The gross cost, gross income, gross margin and production cost of fish weight and protein gain per $\mathrm{kg}$ were evaluated according to the following formulae [27]:

$$
\text { Gross cost }\left(\$ \mathrm{~m}^{-3}\right)=\text { seed cost }+ \text { feed cost }
$$


Gross income $\left(\$ \mathrm{~m}^{-3}\right)=$ the sum of produced species price (P. hypophthalmus and/or O. niloticus)

Gross margin $\left(\$ \mathrm{~m}^{-3}\right)=$ gross income - gross cost

Feed costs $\mathrm{kg}^{-1}$ weight gain $=\mathrm{FCR} \times$ costs of $\mathrm{kg}$ feed

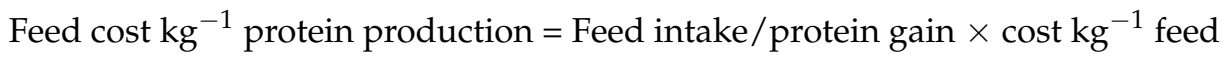

\subsubsection{Diet and Whole-Body Proximate Chemical Compositions}

The chemical composition of the diet and whole-body proximate chemical compositions of the experimental fish were determined according to procedures of [28]. At the end of the experiment, three replicates ( 3 fish species ${ }^{-1} \operatorname{tank}^{-1}$ ) per treatment were killed with overdose of anesthesia ( $5 \mathrm{~g}$ clove oil $\mathrm{L}^{-1}$ ) and used for chemical analysis. Dry matter (DM) was determined after drying the samples in a drying oven $\left(105^{\circ} \mathrm{C}\right)$ for $24 \mathrm{~h}$. Ash was measured following incineration at $550^{\circ} \mathrm{C}$ for $12 \mathrm{~h}$. Crude protein was determined by micro-Kjeldhal method, $(\mathrm{N} \% \times 6.25$ (using Automatic Kjeldahl Analyzer, Model VELP Scientifica UDK 127). Crude fat was assessed by Soxhlet Extractor, Model VELP Scientifica SER 148 (diethyl ether $\left(40-60^{\circ} \mathrm{C}\right)$ ). Crude fiber (CF) in diets was determined after digestion, using $5 \%$ sulphuric acid and 5\% sodium hydroxide for $15 \mathrm{~min}$; the residues were then dried and ashed.

\subsubsection{Organs Somatic Indices}

Viscera and liver were dissected out of nine anaesthetized fish $\left(0.5 \mathrm{~g}\right.$ clove oil $\left.\mathrm{L}^{-1}\right)$ from each species per treatment, weighted individually. The visceral somatic index (VSI) and heptosomatic index (HSI) (were calculated as g/100 g body weight as mentioned afterward:

$$
\begin{aligned}
& \text { VSI }(\%)=100 \times[\text { Viscera weight }(\mathrm{g}) / \text { body weight }(\mathrm{g})] . \\
& \text { HSI }(\%)=100 \times[\text { Liver weight }(\mathrm{g}) / \text { body weight }(\mathrm{g})]
\end{aligned}
$$

\subsection{Statistical Analysis}

The results are presented as mean \pm SE of three replicates. All data were statistically analyzed as a completely randomized design by ANOVA using SPSS (standard version 17.0; SPSS, Chicago, IL, USA). Tukey test was used to compare the differences between means when significant $F$ values were observed at $p \leq 0.05$ levels.

\section{Results}

\subsection{Growth Performance and Production}

For growth performance of farmed striped catfish in monoculture or with Nile tilapia in different polyculture proportions, see Table 2, Figures 1 and 2. The growth of both cultured species was significantly affected by different culture systems. The highest growth criteria (FBW, Gain, SGR and length) were recorded for striped catfish reared in monoculture, followed by striped catfish reared in polyculture with the highest striped catfish stocking percent. The growth performance of Nile tilapia showed no significant difference among different treatments. While it showed an increasing trend in growth criteria with increasing striped catfish percent in polyculture at the same stocking density. The survival of striped catfish decreased significantly in polyculture with high tilapia proportion. Meanwhile, Nile tilapia survival did not affect in different culture techniques. The translation of growth criteria to production per culture units $\left(\mathrm{m}^{-3}\right)$ in different culture systems showed a marked increase in total production, with increasing striped catfish percent. The highest production level was recorded for striped catfish monoculture. 


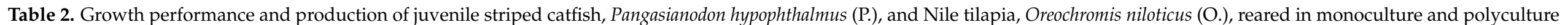
proportions for 14 weeks $(n=3$; means $\pm \mathrm{SE})$.

\begin{tabular}{|c|c|c|c|c|c|c|c|c|c|}
\hline Treat & $\begin{array}{c}\text { Species/ } \\
\text { Proportions }\end{array}$ & $\begin{array}{c}\text { Final Body Weight } \\
\left(\mathrm{g} \mathrm{fish}^{-1}\right)\end{array}$ & $\begin{array}{l}\text { Weight Gain } \\
\left(\mathrm{g} \mathrm{fish}^{-1}\right)\end{array}$ & $\begin{array}{c}\text { Specific Growth Rate } \\
\left(\% \text { day }^{-1}\right)\end{array}$ & $\begin{array}{l}\text { Length } \\
\text { (cm) }\end{array}$ & $\begin{array}{c}\text { Condition } \\
\text { Factor }\end{array}$ & $\begin{array}{l}\text { Survival } \\
(\%)\end{array}$ & $\begin{array}{l}\text { Production } \\
\left(\mathrm{kg} \mathrm{m}^{-3}\right)\end{array}$ & $\begin{array}{c}\text { Total Production } \\
\left(\mathrm{kg} \mathrm{m}^{-3}\right)\end{array}$ \\
\hline $\mathrm{T} 1$ & P. $100 \%$ & $109.50 \pm 5.92^{a}$ & $95.07 \pm 5.84^{\mathrm{a}}$ & $2.07 \pm 0.05^{\mathrm{a}}$ & $25.70 \pm 0.23^{a}$ & $1.01 \pm 0.03^{b}$ & $98.75 \pm 1.25^{\mathrm{a}}$ & $5.41 \pm 0.23^{\mathrm{a}}$ & $5.41 \pm 0.23^{a}$ \\
\hline $\mathrm{T} 2$ & $\begin{array}{l}\text { P. } 25 \% \\
O .75 \%\end{array}$ & $\begin{array}{c}47.01 \pm 3.13^{\mathrm{d}} \\
65.18 \pm 1.97^{\mathrm{bcd}}\end{array}$ & $\begin{array}{c}32.31 \pm 2.93^{\mathrm{d}} \\
50.43 \pm 1.75^{\mathrm{bcd}}\end{array}$ & $\begin{array}{c}1.19 \pm 0.06^{\mathrm{d}} \\
1.52 \pm 0.02^{\text {cde }}\end{array}$ & $\begin{array}{l}17.50 \pm 0.54^{\mathrm{c}} \\
17.10 \pm 0.31^{\mathrm{c}}\end{array}$ & $\begin{array}{l}0.77 \pm 0.04^{\mathrm{b}} \\
1.72 \pm 0.14^{\mathrm{a}}\end{array}$ & $\begin{array}{l}80.00 \pm 0.00^{b} \\
86.67 \pm 3.34^{a}\end{array}$ & $\begin{array}{l}0.47 \pm 0.03^{\mathrm{f}} \\
2.12 \pm 0.01^{\mathrm{c}}\end{array}$ & $2.59 \pm 0.02^{c}$ \\
\hline $\mathrm{T} 3$ & $\begin{array}{l}\text { P. } 50 \% \\
\text { O. } 50 \%\end{array}$ & $\begin{array}{c}83.48 \pm 1.74^{\mathrm{b}} \\
59.44 \pm 1.88^{\mathrm{cd}}\end{array}$ & $\begin{array}{c}68.93 \pm 1.69^{\mathrm{b}} \\
44.85 \pm 1.82^{\mathrm{cd}}\end{array}$ & $\begin{array}{l}1.78 \pm 0.02^{\mathrm{bc}} \\
1.43 \pm 0.03^{\mathrm{de}}\end{array}$ & $\begin{array}{l}23.55 \pm 0.38^{b} \\
16.50 \pm 0.25^{c}\end{array}$ & $\begin{array}{l}0.80 \pm 0.04^{\mathrm{b}} \\
1.97 \pm 0.13^{\mathrm{a}}\end{array}$ & $\begin{array}{l}80.00 \pm 0.00^{b} \\
85.00 \pm 5.00^{a}\end{array}$ & $\begin{array}{l}1.67 \pm 0.04^{\mathrm{cd}} \\
1.27 \pm 0.04^{\mathrm{de}}\end{array}$ & $2.93 \pm 0.00^{c}$ \\
\hline $\mathrm{T} 4$ & $\begin{array}{l}\text { P. } 75 \% \\
\text { O. } 25 \%\end{array}$ & $\begin{array}{l}104.63 \pm 2.85^{a} \\
74.51 \pm 2.02^{b c}\end{array}$ & $\begin{array}{c}90.18 \pm 2.97^{\mathrm{a}} \\
59.86 \pm 2.07^{\mathrm{bc}}\end{array}$ & $\begin{array}{l}2.02 \pm 0.04^{\mathrm{ab}} \\
1.66 \pm 0.03^{\mathrm{cd}}\end{array}$ & $\begin{array}{l}25.73 \pm 0.24^{a} \\
16.50 \pm 0.79^{c}\end{array}$ & $\begin{array}{l}0.98 \pm 0.04^{\mathrm{b}} \\
1.69 \pm 0.04^{\mathrm{a}}\end{array}$ & $\begin{array}{l}86.67 \pm 3.34^{b} \\
85.00 \pm 5.00^{a}\end{array}$ & $\begin{array}{l}3.40 \pm 0.04^{b} \\
0.80 \pm 0.03^{\text {ef }}\end{array}$ & $4.19 \pm 0.02^{b}$ \\
\hline T5 & O. $100 \%$ & $63.32 \pm 5.42^{\mathrm{cd}}$ & $48.87 \pm 5.45^{\mathrm{cd}}$ & $1.50 \pm 0.89$ cde & $17.17 \pm 0.48^{c}$ & $1.02 \pm 0.08^{b c}$ & $91.25 \pm 1.25^{a}$ & $2.88 \pm 0.20^{b}$ & $2.88 \pm 0.20^{c}$ \\
\hline
\end{tabular}

Values (mean \pm SE) in the same column with different superscripts are significantly different using Tukey's test $(p \leq 0.05)$. 
A

B
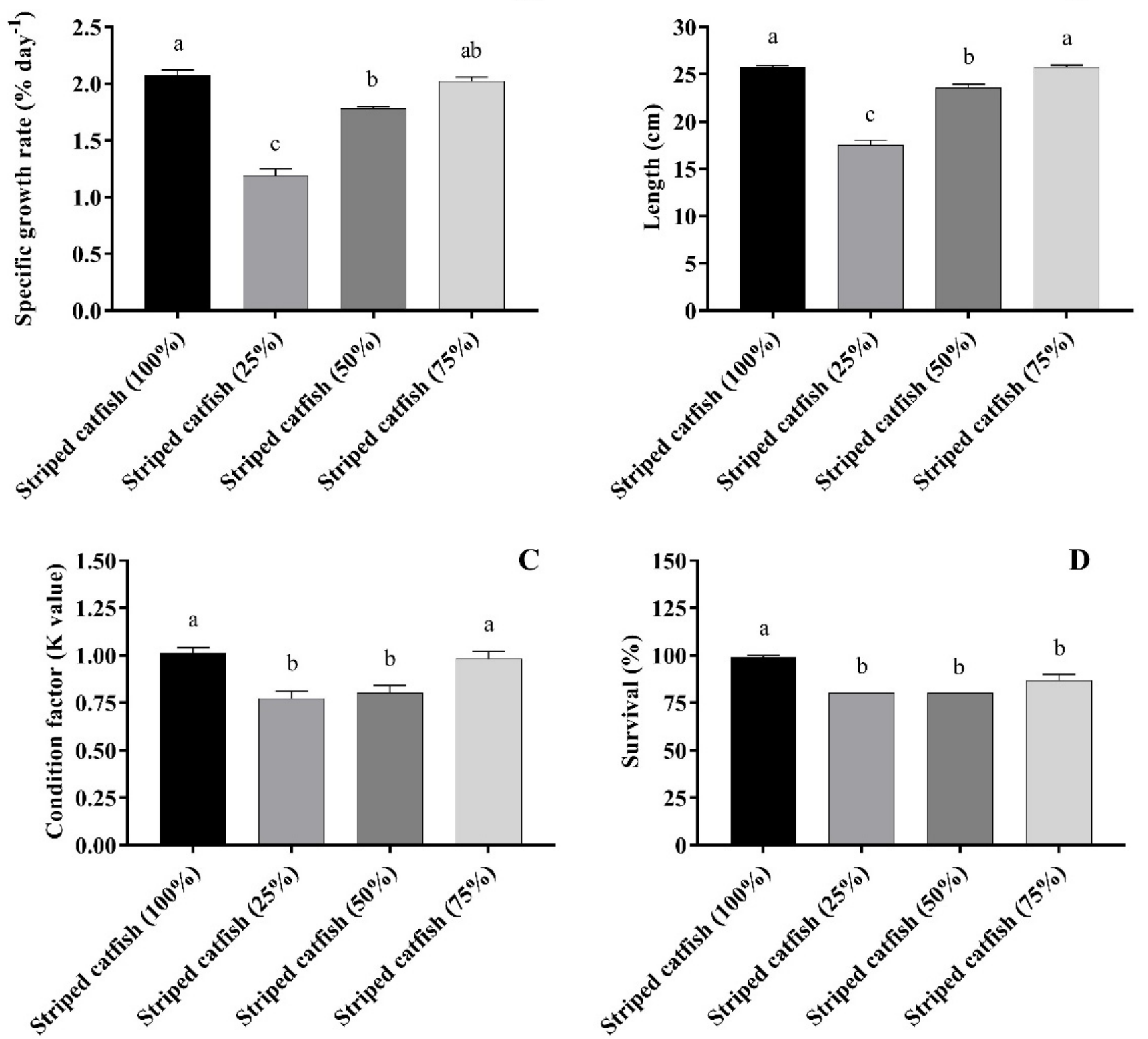

Figure 1. Growth performance (A-C) and survival \% (D) of striped catfish, Pangasianodon hypophthalmus, maintained in monoculture and different polyculture proportions. 
A
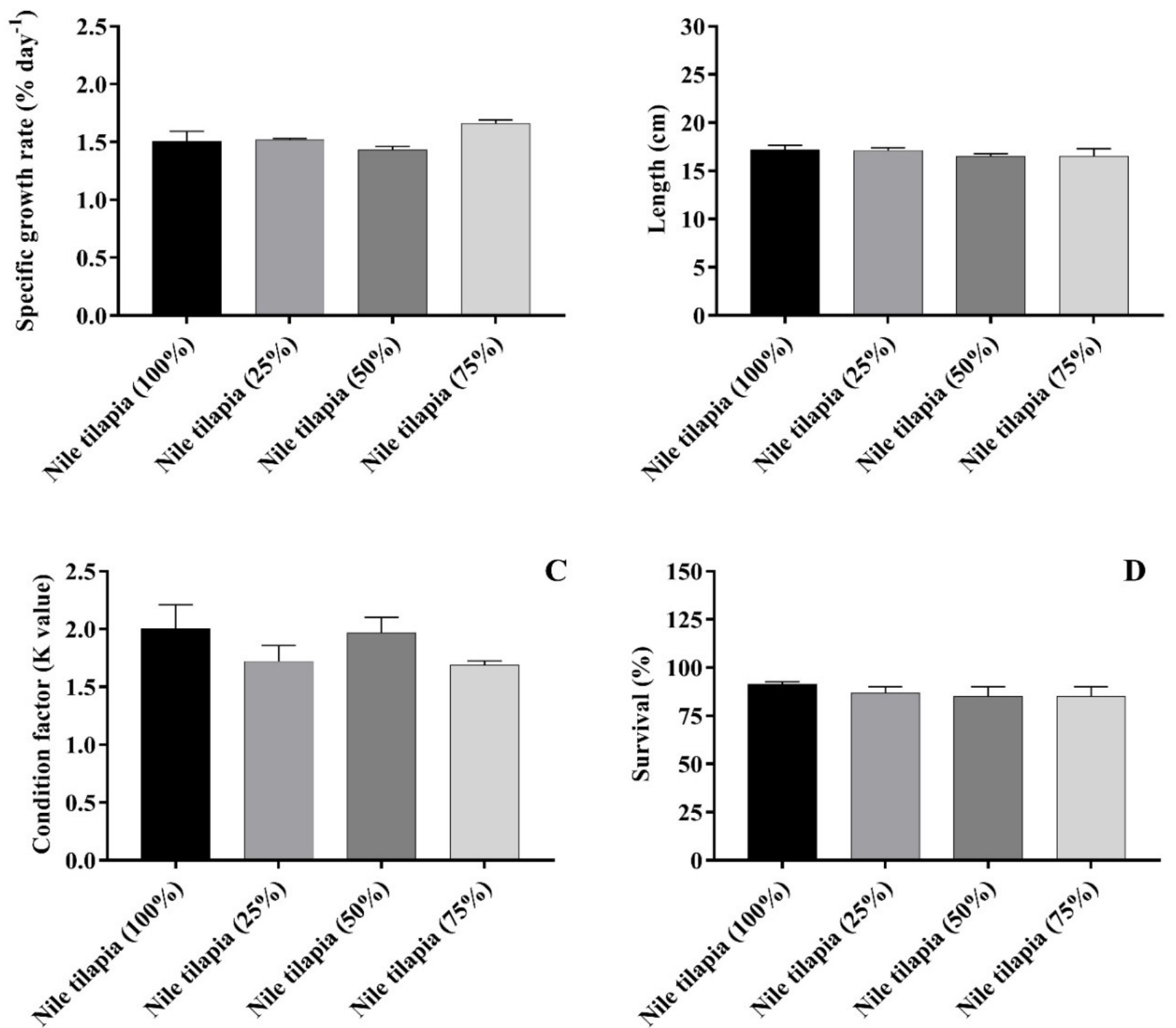

Figure 2. Growth performance (A-C) and survival \% (D) of Nile tilapia, Oreochromis niloticus, maintained in monoculture and different polyculture proportions.

\subsection{Feed Utilization and Somatic Indices}

The feed intake increased significantly in the polyculture proportions (T3 and T4) compared with monoculture of striped catfish (T1) and Nile tilapia (T5). The monoculture of striped catfish significantly attained the best-feed utilization (FCR and PER), compared with different polyculture proportions and monoculture of Nile tilapia (T5). There was no significant difference in hepatosomatic index (\%) and visceral-somatic index (\%) for species in all cultured systems presented in (Table 3). 
Table 3. Feed utilization and somatic indexes of juvenile striped catfish, Pangasianodon hypophthalmus (P.), and Nile tilapia, Oreochromis niloticus (O.), reared in monoculture and polyculture proportions for 14 weeks ( $n=3$; means $\pm \mathrm{SE})$.

\begin{tabular}{|c|c|c|c|c|c|c|}
\hline Treat & $\begin{array}{c}\text { Species/ } \\
\text { Proportions }\end{array}$ & $\begin{array}{l}\text { Feed Intake } \\
\left(\mathrm{g} \mathrm{fish}^{-1}\right)\end{array}$ & FCR & PER & $\begin{array}{c}\text { Hepato-Somatic } \\
\text { Index }(\%)\end{array}$ & $\begin{array}{l}\text { Vesral-Somatic } \\
\text { Index (\%) }\end{array}$ \\
\hline $\mathrm{T} 1$ & P. $100 \%$ & $114.76 \pm 0.08^{\mathrm{cd}}$ & $1.22 \pm 0.08^{b}$ & $2.69 \pm 0.16^{\mathrm{a}}$ & $2.23 \pm 0.14^{\mathrm{ab}}$ & $10.94 \pm 0.85$ \\
\hline $\mathrm{T} 2$ & $\begin{array}{l}\text { P. } 25 \% \\
\text { O. } 75 \%\end{array}$ & $147.98 \pm 9.65^{b c}$ & $1.79 \pm 0.02^{a}$ & $1.11 \pm 0.03^{c}$ & $\begin{array}{l}2.85 \pm 0.10^{\mathrm{ab}} \\
3.02 \pm 0.35^{\mathrm{ab}}\end{array}$ & $\begin{array}{c}10.95 \pm 0.64 \\
9.91 \pm 0.73\end{array}$ \\
\hline T3 & $\begin{array}{l}\text { P. } 50 \% \\
\text { O. } 50 \%\end{array}$ & $161.70 \pm 10.11^{b}$ & $1.42 \pm 0.05^{\mathrm{ab}}$ & $0.90 \pm 0.02^{\mathrm{c}}$ & $\begin{array}{l}1.73 \pm 0.08^{b} \\
3.28 \pm 0.25^{a}\end{array}$ & $\begin{array}{c}9.30 \pm 0.21 \\
10.28 \pm 0.55\end{array}$ \\
\hline $\mathrm{T} 4$ & $\begin{array}{l}\text { P. } 75 \% \\
\text { O. } 25 \%\end{array}$ & $197.86 \pm 1.99^{a}$ & $1.32 \pm 0.02^{\mathrm{ab}}$ & $0.99 \pm 0.03^{c}$ & $\begin{array}{c}2.32 \pm 0.09^{a b} \\
3.41 \pm 0.58^{a}\end{array}$ & $\begin{array}{l}10.99 \pm 0.91 \\
10.02 \pm 1.24\end{array}$ \\
\hline $\mathrm{T} 5$ & O. $100 \%$ & $85.43 \pm 1.55^{\mathrm{d}}$ & $1.76 \pm 0.16^{\mathrm{a}}$ & $1.85 \pm 0.17^{b}$ & $2.25 \pm 0.22^{a}$ & $10.64 \pm 0.33$ \\
\hline
\end{tabular}

Values in the same column with different superscripts are significantly different using Tukey's test $(p \leq 0.05)$.

\subsection{Financial Assessment}

The results of the partial comparative financial assessment depend on cost of feed and seeds per unit of culture systems $\left(\mathrm{m}^{3}\right)$, major variables are presented in Table 4 . The results showed a significant increase in gross cost $\mathrm{m}^{-3}$ in polyculture treatment $(75 \%: 25 \%$ striped catfish and Nile tilapia, respectively).

Table 4. Economical evaluation of juvenile striped catfish, Pangasianodon hypophthalmus (P.), and Nile tilapia, Oreochromis niloticus (O.), reared in monoculture and polyculture proportions for 14 weeks.

\begin{tabular}{|c|c|c|c|c|c|c|}
\hline Treat & Species/Proportions & $\begin{array}{l}\text { Gross Cost } \\
\left(\$ \mathrm{~m}^{-3}\right)\end{array}$ & $\begin{array}{l}\text { Gross Income } \\
\quad\left(\$ \mathrm{~m}^{-3}\right)\end{array}$ & $\begin{array}{l}\text { Net Income } \\
\left(\$ \mathrm{~m}^{-3}\right)\end{array}$ & $\begin{array}{l}\text { Feed Cost } \\
\mathbf{k g}^{-1} \text { Gain }\end{array}$ & $\begin{array}{c}\text { Feed Cost } \mathrm{kg}^{-1} \\
\text { Protein Gain }\end{array}$ \\
\hline $\mathrm{T} 1$ & P. $100 \%$ & $4.17 \pm 0.48^{b}$ & $10.45 \pm 6.71^{\mathrm{a}}$ & $6.21 \pm 7.20^{\mathrm{a}}$ & $0.55 \pm 0.53^{c}$ & $1.22 \pm 0.77^{\mathrm{c}}$ \\
\hline $\mathrm{T} 2$ & P. $25,0.75 \%$ & $4.45 \pm 1.63^{b}$ & $5.00 \pm 0.41^{c}$ & $0.46 \pm 1.21^{\mathrm{c}}$ & $0.81 \pm 0.11^{\mathrm{a}}$ & $2.41 \pm 1.06^{\mathrm{b}}$ \\
\hline $\mathrm{T} 3$ & P. $50,0.50 \%$ & $4.63 \pm 1.55^{b}$ & $5.58 \pm 0.01^{\mathrm{c}}$ & $0.95 \pm 1.54^{\mathrm{cd}}$ & $0.64 \pm 0.33^{b c}$ & $3.02 \pm 1.28^{\mathrm{a}}$ \\
\hline $\mathrm{T} 4$ & P. $75,0.25 \%$ & $5.48 \pm 1.51^{\mathrm{a}}$ & $7.97 \pm 0.39^{b}$ & $2.25 \pm 1.12^{b c}$ & $0.60 \pm 0.16^{b c}$ & $2.54 \pm 0.97^{b}$ \\
\hline $\mathrm{T} 5$ & O. $100 \%$ & $2.97 \pm 0.05^{\mathrm{c}}$ & $5.49 \pm 0.40^{\mathrm{c}}$ & $2.52 \pm 0.39^{b}$ & $0.80 \pm 0.07^{\mathrm{ab}}$ & $1.40 \pm 0.11^{\mathrm{c}}$ \\
\hline
\end{tabular}

Values in the same column with different superscripts are significantly different using Tukey's test $(p \leq 0.05)$, $(n=3$; means \pm SE). Seed cost for 1000 fingerlings of $P$. hypophthalmus $=63.49 \$, 1000$ fingerlings of $O$. nilotcus $=15.87 \$$, Feed cost: $0.52 \$ \mathrm{Kg}^{-1}$.

The monoculture of P. hypophthalmus appeared to have greater profit potential, which showed significantly highest culture system in terms of gross income $\mathrm{m}^{-3}$ and gross margin $\mathrm{m}^{-3}$, followed by the polyculture with higher striped catfish ratio $(75 \%)$. Additionally, the cost of feed per $\mathrm{kg}$ of weight gain significantly increased in the polyculture with higher Nile tilapia percentage (T2) and in Nile tilapia monoculture (T5). On the other hand, the cost of feed per $\mathrm{kg}$ of protein gain significantly decreased in monoculture systems of striped catfish (T1) and of Nile tilapia (T5) more than different polyculture systems.

\subsection{Whole-Body Proximate Chemical Composition}

The whole-body proximate chemical composition of both cultured species was presented in Table 5. The dry matter was significantly different among different cultured species, showing especially a reduction in DM of striped catfish reared in polyculture with low stocking ratios. The crude protein and ash content showed a general significant increase in all Nile tilapia in monoculture or polyculture regimes compared with striped catfish. On the other hand, ether extract increased significantly in striped catfish compared with Nile tilapia. 
Table 5. Whole-body proximate chemical composition of juvenile striped catfish, Pangasianodon hypophthalmus (P.), and Nile tilapia, Oreochromis niloticus (O.), reared in monoculture and different polyculture compositions for 14 weeks $(n=3$; means $\pm \mathrm{SE})$.

\begin{tabular}{cccccc}
\hline Treat & Species/Proportions & $\begin{array}{c}\text { Dry Matter } \\
\mathbf{( \% )}\end{array}$ & $\begin{array}{c}\text { Crude Protein } \\
\mathbf{( \% )}\end{array}$ & $\begin{array}{c}\text { Ether Extract } \\
\mathbf{( \% )}\end{array}$ & $\begin{array}{c}\text { Ash } \\
\mathbf{( \% )}\end{array}$ \\
\hline T1 & $P .100 \%$ & $30.45 \pm 1.40^{\mathrm{ab}}$ & $45.05 \pm 0.95^{\mathrm{c}}$ & $38.35 \pm 2.22^{\mathrm{ab}}$ & $8.63 \pm 0.48^{\mathrm{d}}$ \\
$\mathrm{T} 2$ & $P .25 \%$ & $27.23 \pm 0.15^{\mathrm{b}}$ & $49.89 \pm 1.44^{\mathrm{bc}}$ & $36.82 \pm 0.36^{\mathrm{ab}}$ & $9.94 \pm 0.35^{\mathrm{cd}}$ \\
& $0.75 \%$ & $28.92 \pm 0.09^{\mathrm{ab}}$ & $55.56 \pm 0.15^{\mathrm{ab}}$ & $29.29 \pm 0.27^{\mathrm{cd}}$ & $13.03 \pm 0.95^{\mathrm{b}}$ \\
$\mathrm{T} 3$ & $P .50 \%$ & $30.49 \pm 0.02^{\mathrm{ab}}$ & $50.98 \pm 2.75^{\mathrm{abc}}$ & $39.44 \pm 1.87^{\mathrm{a}}$ & $9.99 \pm 0.47^{\mathrm{cd}}$ \\
& $0.50 \%$ & $30.92 \pm 0.61^{\mathrm{ab}}$ & $54.47 \pm 0.26^{\mathrm{ab}}$ & $32.18 \pm 1.57^{\mathrm{abc}}$ & $12.66 \pm 0.16^{\mathrm{bc}}$ \\
T4 & $P .75 \%$ & $33.36 \pm 1.84^{\mathrm{a}}$ & $47.47 \pm 0.26^{\mathrm{bc}}$ & $35.96 \pm 0.29^{\mathrm{ab}}$ & $9.83 \pm 0.51^{\mathrm{cd}}$ \\
$\mathrm{T} 5$ & $O .25 \%$ & $26.30 \pm 0.68^{\mathrm{b}}$ & $59.58 \pm 2.91^{\mathrm{a}}$ & $23.62 \pm 2.92^{\mathrm{d}}$ & $16.57 \pm 0.26^{\mathrm{a}}$ \\
\hline
\end{tabular}

Values in the same column with different superscripts are significantly different using Tukey's test $(p \leq 0.05)$.

\section{Discussion}

Aquaculture investments are simulated based on a set of production parameters and estimated costs of investment which prospect to be applied to more commercial-scale operations in developing countries. Monoculture and polyculture are the most common aquaculture systems made for fish production in many countries of the world $[8,18]$. In each aquaculture system, the growth rate which determines the total production of the aquaculture system is affected by several factors including, intraspecific and interspecific interaction. Furthermore, fish growth is affected by many factors such as seasonality, availability of food and oxygen, stocking density, age and aquaculture system $[6,29]$. Previous studies regarding the polyculture of striped catfish and Nile tilapia showed that both species may be co-farmed without affecting growth performance or survival $[9,30]$. The most important issue in this matter is to determine optima proportions of both species in polyculture system, which will be the main aquaculture practice in Africa, in order to maximize growth performance, productivity and revenue. However, this is a matter still to be determined. Thus, the evaluation of best proportions of polyculture system has a great role in maximizing the total production and financial revenue.

Present results reveal that higher growth rate and feed utilization of striped catfish are found in monoculture. In the same context, the monoculture of striped catfish $P$. hypophthalmus has a higher fish biomass production than the polyculture with silver carp, though with low economic profitability [31]. In the present study, SGR of striped catfish reach 2.07 and 1.19 in monoculture and polyculture with low striped catfish proportion, respectively. Thus, SGR of striped catfish reared in monoculture or in highly proportioned polyculture was higher than SGR in polyculture with rohu, Labeo rohita [32]. SGR values in the present study surpass other previous experiments on striped catfish [32,33]. The present findings reveal that the relations between cultivated striped catfish with Nile tilapia in a polyculture system, growth and yield parameters are in close agreement with many reports on other catfish species, as tilapia comes after striped catfish in growth performance [10,34-37].

The improvements of growth performance of striped catfish in the present study, as its stocking density increases, reaching up to monoculture ratio $(100 \%)$ may be due to the decrease of competition between Nile tilapia and striped catfish (intra-species competition) for feeding [13]. the same for the better environmental conditions and feeding habits in the same species $[38,39]$. In addition, the initial stocking weight of both species used in the present study is similar, however, the large tilapia groups $(75 \%$ and $50 \%$ of tank proportions) suppress the growth of striped catfish $\left(\mathrm{T}_{2}\right.$ and $\left.\mathrm{T}_{3}\right)$. This may be attributed to that the larger tilapia group is, the probability of fighting for food and presence of territorially based hierarchy leading to social instability increase [40,41]. On the other hand, the polyculture of striped catfish with silver carp was better than monoculture [14]. This 
difference with the present study may be due to different feeding habits (filter feeders and omnivorous), density and species proportions in both experiments.

The survival of striped catfish in monoculture was $98.75 \%$, it decreased significantly in polyculture with high tilapia proportions. However, survival of tilapia showed no significant difference among different polyculture treatments in the present study. These results suggested that striped catfish did not pray on tilapia, especially if they had equal initial size, thus, it is possible to farm both species. Therefore, survival of striped catfish in monoculture (90\%) was higher than in polyculture [42]. Moreover, ranges of striped catfish survival were $94-96 \%$ in ponds, [33], $92-95 \%$ in cages, [43] and close to $100 \%$ in cemented cisterns [44]. The present rates of survival in polyculture treatments were lower than those reported in polyculture of striped catfish (95-97\%) and tilapia (91-93\%) [9]. This might be due to high stocking density in the present study compared with other polyculture proportions [9] and the difference in species proportions [45].

In addition, the production of each water unit in different culture systems was significantly different in the present study. The highest total production was in monoculture of striped catfish $\left(\mathrm{T}_{1}, 5.41 \mathrm{~kg} \mathrm{~m}^{-3}\right)$ followed by polyculture with high striped catfish proportions $\left(\mathrm{T}_{4}, 4.19 \mathrm{~kg} \mathrm{~m}^{-3}\right)$. Being the most detrimental factors in total production of culture systems, the growth performance and survival were higher, in the present findings, in monoculture of striped catfish than in polyculture with tilapia. The final harvested biomass of each $\mathrm{m}^{-3}$ of water was closely related to survival rate [46]. Thus, the monoculture of striped catfish resulted in higher productivity compared to polyculture with different culture species [16,47]. Moreover, the production of striped catfish/tilapia polyculture in the present study recorded higher productivity $\left(2.59-4.19 \mathrm{~kg} \mathrm{~m}^{-3}\right)$ than polyculture at stocking ratio $5: 3$ in earthen ponds $\left(0.83 \mathrm{~kg} \mathrm{~m}^{-3}\right)$ [9].

The determination of financial benefit in the present study of the monoculture and polyculture of striped catfish with Nile tilapia was evaluated, given the main inputs, including feeding and stocking seeds cost, which were the most significant factors influencing fish production costs [48]. The present findings revealed that the highest gross income, gross margin and least cost of weight and protein gain production per $\mathrm{kg}$ were recorded for monoculture of striped catfish, followed by polyculture with high striped catfish proportions. This contrasted with the polyculture of striped catfish and other species (Carps, cattala and freshwater prawn), it was more economically efficient even if it recorded low production volume $[16,30,31,34]$. These results relied on the economic fact that not all fish species have the same selling price [49]. Therefore, polyculture might yield higher profits than monoculture [50]. In the present study, the selling price of Nile tilapia and striped catfish is nearly similar on the local market, therefore, the revenue was improved with monoculture of striped catfish, due to higher growth and survival rates (total production) in monoculture than in polyculture.

Regarding the proximate chemical composition of culture striped catfish and Nile tilapia in the different studied systems, the results did not reveal any significant differences of protein, ether extract or ash among studied treatments, in respect to species. In general, the ether extract content of striped catfish was higher than Nile tilapia and ether extract was inversely proportional to the crude protein content. Striped catfish had a high lipid content ( $12 \%$ on wet weight basis), however, most lipids were found in the viscera and abdominal muscles [51]. Furthermore, striped catfish showed contents of protein (23-26\%) and lipids (11-14\%) in wet weight bases [52].Striped catfish could be classified as a medium-fat to highly fat fish $[51,53]$. The tilapia proximate composition showed similar chemical contents (14-17\% protein content and $6-10 \%$ lipids on wet weight basis) with previous studies [54-56].

\section{Conclusions}

As the inclusion of striped catfish, P. hypophthalmus, in the African freshwater aquaculture is just taking its first steps, to maintain the sustainable production development of inland aquaculture, the study of farming striped catfish with the endogenous specie Nile 
tilapia, O. niloticus, was evaluated to provide sufficient scientific information on polyculture proportions. The results clearly proved that better growth performance, feed utilization and survival of striped catfish were recorded in monoculture and polyculture with low Nile tilapia proportion. The highest total production and revenue were obtained in monoculture of striped catfish. Regarding Nile tilapia, growth and survival showed no difference among different studied treatments. Thus, ecologically, the farming of tilapia and striped catfish is applicable, however, for higher production and economic profit, the monoculture of striped catfish is recommended.

Author Contributions: Conceptualization, T.M.S., E.A.O. and A.A.M.N.; data curation, B.W.A. and H.S.K.; formal analysis, A.T.M. and B.W.A.; funding acquisition, A.T.M. and B.W.A.; investigation, B.W.A.; methodology, B.W.A.; project administration, E.A.O. and A.A.M.N.; resources, A.T.M. and B.W.A.; software, A.T.M. and H.S.K.; supervision, T.M.S. and E.A.O.; validation, A.T.M., B.W.A., T.M.S., E.A.O., A.A.M.N. and H.S.K.; visualization, A.T.M. and H.S.K.; writing-original draft, A.T.M., B.W.A. and H.S.K.; writing-review and editing, A.T.M. and H.S.K. All authors have read and agreed to the published version of the manuscript.

Funding: The authors extend their appreciation to the Deputyship for Research \& Innovation, Ministry of Education in Saudi Arabia for funding this research work through the project number IFT20179.

Institutional Review Board Statement: Institutional Animal Care and Use Committee of Alexandria University (IACUC) approved experimental protocols (Approval No AU: 14200721310).

Informed Consent Statement: Not applicable.

Data Availability Statement: The data are available upon reasonable request.

Conflicts of Interest: The authors declare no conflict of interest.

\section{References}

1. FAO. The State of World Fisheries and Aquaculture 2020. Sustainability in Action; FAO: Rome, Italy, 2020. [CrossRef]

2. Ahmed, M.; Lorica, M.H. Improving developing country food security through aquaculture development-lessons from Asia. Food Policy 2002, 27, 125-141. [CrossRef]

3. Pradeepkiran, J.A. Aquaculture role in global food security with nutritional value: A review. Transl. Anim. Sci. 2019, 3, 903-910. [CrossRef]

4. El-Sayed, A.-F.M. Tilapia Culture; CABI Publishing: Willingford, Oxfordshire, UK, 2006.

5. Behera, B.; Pradhan, P.; Swaminathan, T.; Sood, N.; Paria, P.; Das, A.; Verma, D.; Kumar, R.; Yadav, M.; Dev, A. Emergence of tilapia lake virus associated with mortalities of farmed Nile tilapia Oreochromis niloticus (Linnaeus 1758) in India. Aquaculture 2018, 484, 168-174. [CrossRef]

6. Mehrim, A.; Refaey, M.; Khalil, F.; Shaban, Z.E. Impact of Mono-and Polyculture Systems on Growth Performance, Feed Utilization, and Economic Efficiency of Oreochromis niloticus, Mugil cephalus, and Mugil capito. J. Anim. Poult. Prod. 2018, 9 , 393-400. [CrossRef]

7. Jayanthi, M.; Thirumurthy, S.; Samynathan, M.; Manimaran, K.; Duraisamy, M.; Muralidhar, M. Assessment of land and water ecosystems capability to support aquaculture expansion in climate-vulnerable regions using analytical hierarchy process based geospatial analysis. J. Environ. Manag. 2020, 270, 110952. [CrossRef]

8. Allam, B.W.; Khalil, H.S.; Mansour, A.T.; Srour, T.M.; Omar, E.A.; Nour, A.A.M. Impact of substitution of fish meal by high protein distillers dried grains on growth performance, plasma protein and economic benefit of striped catfish (Pangasianodon hypophthalmus). Aquaculture 2020, 517, 734792. [CrossRef]

9. Mahmud, S.; Ali, M.L.; Alam, M.A.; Rahman, M.M.; Jørgensen, N.O. Effect of probiotic and sand filtration treatments on water quality and growth of tilapia (Oreochromis niloticus) and pangas (Pangasianodon hypophthalmus) in earthen ponds of southern Bangladesh. J. Appl. Aquac. 2016, 28, 199-212. [CrossRef]

10. Hossain, M.A.; Islam, M.S. Optimization of stocking density of freshwater prawn Macrobrachium rosenbergii (de Man) in carp polyculture in Bangladesh. Aquac. Res. 2006, 37, 994-1000. [CrossRef]

11. Phuong, N.; Oanh, D. Striped catfish aquaculture in Vietnam:a decade of unprecedented development. In Success Stories in Asian Aquaculture; De Silva, S., Davy, F., Eds.; Springer: Dordrecht, The Netherlands, 2010; pp. 131-147.

12. Offem, B.O.; Ikpi, G.U.; Ayotunde, E.O. Effect of stocking size of the predatory African catfish (Heterobranchus longifilis V.) on the growth performance of Nile Tilapia (Oreochromis niloticus L.) in pond culture. Int. J. Fish. Aquac. 2009, 1, 38-43.

13. Shafiullah, M.; Siddique, M.A.B.; Rahman, M.S.; Mahalder, B.; Ali, A.; Rahmatullah, S. Effects of Different Stocking Ratios on the Production and Survival of Indigenous Carps and Pangas (Pangasius hypophthalmus) in a Pond System. Master's Thesis, Department of Aquaculture, Bangladesh Agricultural University, Mymensingh, Bangladesh, 2003. 
14. Sarkar, M.; Khan, S.; Haque, M.; Haq, M. Evaluation of growth and water quality in pangasiid catfish (Pangasius hypophthalmus) monoculture and polyculture with silver carp (Hypophthalmichthys molitrix). J. Bangladesh Agric. Univ. 2006, 4, 339-346.

15. Khan, S.; Hossain, M.; Haque, M. Effects of feeding schedule on growth, production and economics of pangasiid catfish (Pangasius hypophthalmus) and silver carp (Hypophthalmichthys molitrix) polyculture. J. Bangladesh Agric. Univ. 2009, 7, 175-181. [CrossRef]

16. Islam, M.S.; Huq, K.A.; Rahman, M.A. Polyculture of Thai pangus (Pangasius hypophthalmus, Sauvage 1878) with carps and prawn: A new approach in polyculture technology regarding growth performance and economic return. Aquac. Res. 2008, 39, 1620-1627. [CrossRef]

17. Husain, T.K.; Mulyo, J.H.; Jamhari, J. Analisis Perbandingan Keuntungan dan Risiko Usaha Perikanan Rakyat Sistem Monokultur dan Polikultur di Kabupaten Pangkep. Agro Ekon. 2016, 27, 136-149. [CrossRef]

18. Sudirman, A.; Rahardjo, S.; Rukmono, D. Economical analysis of Polyculture of Catfish and Tilapia Fishi Biofloc System. Int. J. Eng. Sci. 2020, 9, 1-7.

19. Ali, M.; Islam, M.; Begum, N.; Suravi, I.; Mia, M.; Kashem, M. Effect of Monoculture and Polyculture Systems on Growth and Production of Fishes in Seasonal Waterbodies of Haor Villages, Sunamganj District. J. Sci. Res. 2017, 9, 307-316. [CrossRef]

20. FAO. The State of World Fisheries and Aquaculture; FAO: Rome, Italy, 2018.

21. Azim, M.; Verdegem, M.; Mantingh, I.; Van Dam, A.; Beveridge, M. Ingestion and utilization of periphyton grown on artificial substrates by Nile tilapia, Oreochromis niloticus L. Aquac. Res. 2003, 34, 85-92. [CrossRef]

22. Ali, H.; Haque, M.M.; Belton, B. Striped catfish (Pangasianodon hypophthalmus, Sauvage, 1878) aquaculture in Bangladesh: An overview. Aquac. Res. 2013, 44, 950-965. [CrossRef]

23. Belton, B.; Van Asseldonk, I.J.M.; Bush, S.R. Domestic crop booms, livelihood pathways and nested transitions: Charting the implications of Bangladesh's Pangasius boom. J. Agrar. Chang. 2017, 17, 694-714. [CrossRef]

24. Haque, R.I.; Azam, M.R.; Ullah, M.A. Production of Stinging Catfish (Heteropneustes fossilis) in different stock-ing densities with GIFT (Oreochromis niloticus) and Thai Sharpunti (Bar). J. Fish. Life Sci. 2018, 3, 9-15.

25. Tang, Q. Modification of the Ricker stock recruitment model to account for environmentally induced variation in recruitment with particular reference to the blue crab fishery in Chesapeake Bay. Fish. Res. 1985, 3, 13-21. [CrossRef]

26. NRC. Nutrient Requirements of Fish and Shrimp; National Academies Press: Washington, DC, USA, 2011.

27. Khalil, H.S.; Mansour, A.T.; Goda, A.M.A.; Omar, E.A. Effect of selenium yeast supplementation on growth performance, feed utilization, lipid profile, liver and intestine histological changes, and economic benefit in meagre, Argyrosomus regius, fingerlings. Aquaculture 2019, 501, 135-143. [CrossRef]

28. AOAC. Association of Official Analytical Chemists. Off. Methods Anal. Assoc. Off. Anal. Chem. Gaithersburg MD USA 2000, 260-267.

29. M'balaka, M.; Kassam, D.; Rusuwa, B. The effect of stocking density on the growth and survival of improved and unimproved strains of Oreochromis shiranus. Egypt. J. Aquat. Res. 2012, 38, 205-211. [CrossRef]

30. Azad, M.; Rahman, M.; Rahman, Z. Polyculture of carp, tilapia and pangas using low cost inputs. Pak. J. Biol. Sci. $2004,7,11$.

31. Sarkar, M.; Khan, S.; Haque, M. Production and economic return in pangasiid catfish (Pangasius hypophthalmus) monoculture and polyculture with silver carp (Hypophthalmichthys molitrix) in farmers' ponds. Bangladesh J. Fish. Res. 2005, 9, 111-120.

32. Sayeed, M.; Hossain, G.; Mistry, S.; Huq, K. Growth performance of thai pangus (pangasius hypophthalmus) in polyculture system using different supplementary feeds. Univ. J. Zool. Rajshahi Univ. 2008, 27, 59-62. [CrossRef]

33. Haroon, A.; Shanta, M. Culture Feasibility Study of Pangasius Sutchi (Fowler) at a High Density in the Upazilla Parishad's Pond; Bangladesh Fisheries Research Institute, Chandpur and Bangladesh Agricultural Research Council: Dhaka, Bangladesh, 2001; pp. 63-65.

34. Limbu, S.M.; Shoko, A.P.; Lamtane, H.A.; Shirima, E.D.; Kishe-Machumu, M.A.; Mgana, H.F.; Mgaya, Y.D. Effect of initial stocking size of the predatory African sharptooth catfish (Clarias gariepinus) on recruits, growth performance, survival and yield of mixed-sex Nile tilapia (Oreochromis niloticus) in concrete tank culture system. Int. Aquat. Res. 2015, 7, 63-73. [CrossRef]

35. Asadujjaman, M.; Azad, M.A.K.; Ali, M.R.; Hossain, M.A. Optimization of stocking density for Azolla based carp polyculture pond. J. Fish. Aquac. Stud. 2016, 4, 273-279.

36. Rahman, M.T.; Nielsen, R.; Khan, M.A. Agglomeration externalities and technical efficiency: An empirical application to the pond aquaculture of Pangas and Tilapia in Bangladesh. Aquac. Econ. Manag. 2019, 23, 158-187. [CrossRef]

37. Setiadi, E.; Taufik, I. Polyculture of Giant Freshwater Prawn, Macrobrachium rosenbergii, and Nilem Carp, Osteochilus hasselti, Cultured in Recirculation System Using Biofiltration. In Proceedings of the E3S Web of Conferences, Purwokerto, Indonesia, 1 August 2018; Volume 47, p. 02005.

38. Solomon, J.R.; Boro, S.G. Survival rate in polyculture of Catfish Heteroclarias/Tilapia (Oreochromis niloticus), fed $2 \%$ body weight. N. Y. Sci J. 2010, 3, 68-78.

39. Uddin, M.S.; Rahman, S.S.; Azim, M.E.; Wahab, M.A.; Verdegem, M.J.; Verreth, J.A. Effects of stocking density on production and economics of Nile tilapia (Oreochromis niloticus) and freshwater prawn (Macrobrachium rosenbergii) polyculture in periphytonbased systems. Aquac. Res. 2007, 38, 1759-1769. [CrossRef]

40. Nelissen, M.H. Does body size affect the ranking of a cichild fish in a dominance hierarchy? J. Ethol. 1992, 10, 153-156. [CrossRef]

41. Gonçalves-de-Freitas, E.; Teresa, F.B.; Gomes, F.S.; Giaquinto, P.C. Effect of water renewal on dominance hierarchy of juvenile Nile tilapia. Appl. Anim. Behav. Sci. 2008, 112, 187-195. [CrossRef] 
42. Haldar, G.; Jahan, D. Polyculture of Pangus with Carp at Low Density in a Farmer's Pond; Bangladesh Fisheries Research Institute, Chandpur and Bangladesh Agricultural Research Council: Dhaka, Bangladesh, 2001; pp. 66-74.

43. Azimuddin, K.; Hossain, M.; Wahab, M.; Noor, J. Effect of stocking density on the growth of Thai pangas, Pangasius sutchi (Fowler) in net cage fed on formulated diet. Bangladesh J. Fish. Res. 1999, 3, 173-180.

44. Haroon, A.; Hossain, M. Studies on the Polyculture of Pangasius Sutchi (Fowler) in Cemented Cisterns; Bangladesh Fisheries Research Institute, Chandpur and Bangladesh Agricultural Research Council: Dhaka, Bangladesh, 2001; pp. 12-18.

45. Gu, D.E.; Yu, F.D.; Hu, Y.C.; Wang, J.W.; Xu, M.; Mu, X.D.; Yang, Y.X.; Luo, D.; Wei, H.; Shen, Z.X. The species composition and distribution patterns of non-native fishes in the main rivers of South China. Sustainability 2020, 12, 4566. [CrossRef]

46. Rosmawati, M.A. Survival and growth of African catfish (Clarias sp.) seed in recirculation systems with different densities. J. Pertan. 2011, 2, 36-47.

47. Srrps, D.; Iswanto, B. Productivity and profitability of selection of catfish (Clarias gariepinus) aquaculture in rearing in soil ponds. Media Akuakultur. 2016, 11, 11-17.

48. Ali, H.; Rahman, M.E.; Murshed-e-Jahan, K.; Dhar, G.C. Production economics of striped catfish (Pangasianodon hypophthalmus, Sauvage, 1878) farming under polyculture system in Bangladesh. Aquaculture 2018, 491, 381-390. [CrossRef]

49. Moray, J.C.; Saerang, D.P.E.R.T. Determination of the selling price with cost plus pricing uses the full costing approach on ud gladys bakery. J. EMBA 2014, 2, 1272-1283.

50. Husain, T.K.; Mulyo, J.H.; Jamhari, J. Comparative analysis of the benefits and risks of smallholder monoculture and polyculture fisheries business in Pangkep Regency. Agro Ekon. 2016, 27, 136-149. [CrossRef]

51. Thammapat, P.; Raviyan, P.; Siriamornpun, S. Proximate and fatty acids composition of the muscles and viscera of Asian catfish (Pangasius bocourti). Food Chem. 2010, 122, 223-227. [CrossRef]

52. Monalisa, K.; Islam, M.; Khan, T.; Abdullah, A.; Hoque, M. Comparative study on nutrient contents of native and hybrid Koi (Anabas testudineus) and Pangas (Pangasius pangasius, Pangasius hypophthalmus) fish in Bangladesh. Int. Food Res. J. 2013, 20, 791-797.

53. Haard, N.F. Control of chemical composition and food quality attributes of cultured fish. Food Res. Int. 1992, 25, 289-307. [CrossRef]

54. Mansour, A.T.; Esteban, M.Á. Effects of carbon sources and plant protein levels in a biofloc system on growth performance, and the immune and antioxidant status of Nile tilapia (Oreochromis niloticus). Fish Shellfish Immunol. 2017, 64, 202-209. [CrossRef] [PubMed]

55. Mansour, A.; Omar, E.; Srour, T.; Yousef, M. Effect of three natural phytochemicals supplementation on growth performance, testosterone level and feed utilization of Nile tilapia (Oreochromis niloticus). Aquac. Nutr. 2018, 24, 408-415. [CrossRef]

56. Khalila, H.; Fayed, W.; Mansour, A.; Srour, T.; Omar, E.; Darwish, S.; Nour, A. Dietary Supplementation of Spirulina, Arthrospira platensis, with Plant Protein Sources and their Effects on Growth, Feed Utilization and Histological Changes in Nile Tilapia, Oreochromis niloticus. J. Aquac. Res. Dev. 2018, 9, 2. [CrossRef] 